\title{
Laser heating induced plastic deformation in a pre-elastic-stretched titanium alloy strip
}

\author{
Chen-Wu Wu *, Chen-Guang Huang, Guang-Nan Chen, Ming-Xing Wang \\ Institute of Mechanics, Chinese Academy of Sciences, No. 15 Beisihuanxi Road, Beijing 100190, PR China
}

\section{A R T I C L E I N F O}

\section{Article history:}

Received 24 April 2012

Received in revised form

25 May 2012

Accepted 25 May 2012

Available online 14 June 2012

Keywords:

Pre-elastic-stretched strip

Laser irradiation

Plastic deformation

\begin{abstract}
A B S T R A C T
Experiment was carried out to reveal the plastic deformation of pre-elastic-stretched titanium alloy strip partially subjected to localized heating via Laser beam irradiation. The temperature profile in the strip under Laser beam irradiation was computed with finite element method. The strain redistribution and localized plastic deformation were analyzed by a one-dimensional model. The results indicate that both the pre-strain level and the ratio of the length of heated range to the total effective length of the strip influence obviously the final plastic strain.
\end{abstract}

(c) 2012 Elsevier Ltd. All rights reserved.

\section{Introduction}

Temperature elevation could improve the ductility of most materials by decreasing the resistance to dislocation sliding etc. Therefore many manufacturing processes adopted heating in metallic alloy plate forming [1-3]. Attracted by the advantages of Laser as a heating source, many investigations have been conducted on the forming mechanism by pure laser heating $[4,5]$. In these processes, the thermal loadings are commonly exerted on the work pieces no latter than the mechanical loadings or even without the mechanical loadings. A new process technique of Laser aided pre-stressed forming was reported to be successfully applied to sheet forming, in which the plastic deformation is developed in a pre-elastic-bended panel by Laser beam heating [6]. In such a process, the non-uniform heating is used to induce plastic deformation in these discrete small regions of the pre-elastic-stretched panel skin. This technique may make full use of the controllability of the heating source to obtain plastic deformation at specific regions [7]. Although the mechanism on the plastic deformation under the thermo-mechanical circumstance need more detailed description.

Actually, a great many of pre-stressed structures may practice non-uniform temperature elevation due to external localized heating $[8,9]$. In a structure subjected to tensile stress high enough, the plastic strain will be developed and concentrated

\footnotetext{
* Corresponding author. Tel.: +86 1082544271 ; fax: +86 1062561284

E-mail addresses: chenwuwu@imech.ac.cn, wcw_on@yahoo.com.cn (C.W.Wu).
}

on some region wherein the material weakening arises due to the defects or other disturbances, which will in turn enhance the material weakening. The plastic deformation concentration of the materials under pure mechanical loading was extensively discussed by many literatures. For instance, Doghri and Billardon [10] investigated the localized plastic deformation for rate-independent plasticity, Haddag et al. [11] set up a large deformation anisotropic elastic-plastic model to describe the plastic strain concentration, Kobayashi [12] analyzed this phenomenon via a proposed theory of ultrasonic wave velocity, Bychkov and Karpinskii [13] studied the localized plastic behavior in a thermoviscoplastic rod in dynamic tension by the methods of linear perturbation analysis. Considering that fact that temperature elevation is also a kind of disturbance, the plastic strain concentration of the structure subjected to both thermal and mechanical loadings also attracted many interests. In particular, Elfmark [14] revealed that plastic instability shall develop in a hot tensile test specimen, in which the specimen was assumed to be evenly heated. Bychkov and Karpinskii $[15,16]$ investigated the plastic deformation concentration phenomena in a tensioned thermoviscoplastic rod heated by an electric current and found out that the action of the joule heat developed by the alternating electric current influenced obviously the plastic behavior in the rod. Fressengeas and Molinari [17] studied the effects of thermal softening on ductility of the material with a one-dimensional model for uniaxial tension and described the dynamic growth of the voids and the adiabatic decrease of ductility. Benallal and Bigoni [18] investigated the influences of temperature elevation due to thermo-mechanical coupling effect on the plasticity of some inelastic material. Basically, these researches mainly 
focused on the plastic deformation behavior in the rod subjected to uniform heating or under the action of the heat converted from the strain energy due to thermo-mechanical coupling effect.

This work is focused on such a problem to describe the plastic deformation of pre-elastic-stretched titanium alloy strip subjected to Laser heating. First, the experiment was carried out to verify the plastic deformation arising in a pre-elastic-stretched strip sample subjected to local heating via Laser irradiation. Then, the temperature characteristic was analyzed numerically. Finally, the strain redistribution behavior was discussed by a one-dimensional model and the effects of the various parameters on the strain redistribution and final plastic strain were investigated.

\section{Experiments}

The equipments as shown in Fig. 1(a) were used to stretch the strip and locally heat the strip. The Nd-YAG Laser was adopted to partially irradiate the sample to realize the non-uniform temperature elevation, of which the effective power is about $48 \mathrm{~W}$ evenly distributed over the circular region of radius $3 \mathrm{~mm}$. The initial strain was controlled to be low enough to ensure that no plastic strain arises in the pre-elastic-stretched sample.

The geometry of the specimen is shown in Fig. 1(b), of which the thermo-mechanical parameters are listed in Table 1 by referencing to the available database and literatures [19,20]. In Fig. 1(b), the test points $\mathrm{CH} 1$ and $\mathrm{CH} 2$ are located on the back side of the specimen relative to the irradiated surface. The virtual test point $\mathrm{CH} 3$ represents

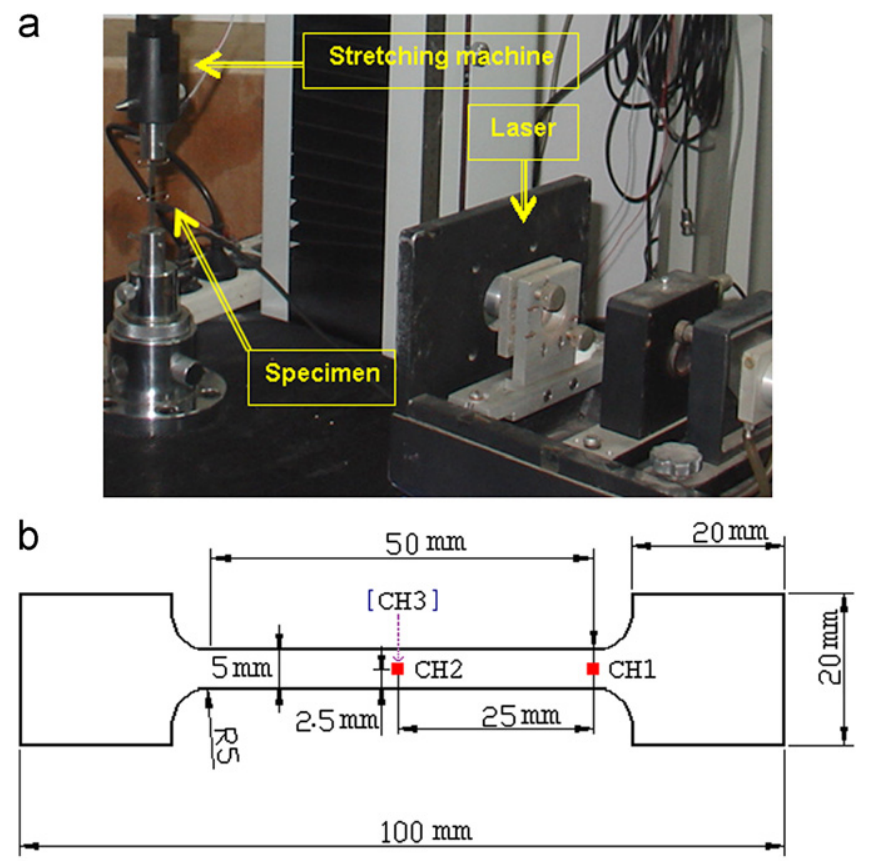

Fig. 1. (a) Photo of the experimental equipment and (b) sketch of the specimen. the center of the irradiated region, for which the temperature history will be investigated only by numerical simulation.

The experiment mainly includes such three stages. That is:

1st step: Stretch the sample to some level of elastic deformation and keep that elastic deformation;

2nd step: heat the segment of little length to some temperature level and then remove the heating via shutting down the laser;

3rd step: Unload the mechanical stretch after the sample is cooled to be identical to the ambient.

Fig. 2 presents the temperature histories of the test points $\mathrm{CH} 1$ and $\mathrm{CH} 2$, which shows that the temperature at the point $\mathrm{CH} 2$ changes very slightly throughout the test. This means that only the heat conduction within the central strip of length about $50 \mathrm{~mm}$ needs to be considered in the computation to obtain accurate enough theoretical description of the temperature profile in the specimen.

The front view of the final deformation of the sample is shown in Fig. 3, which indicates an obvious necking around the center of the heated region.

The side view profile of the necked region is magnified shown in Fig. 4(a), in which one can see that the plastic strain concentrates around the center of the heated segment. Further, it is indicated that there exists a trapezoid plastic deformation zone with the bottom base of length $4.3 \mathrm{~mm}$ being located at the heated side of the specimen while the top base of length $2.3 \mathrm{~mm}$ at the other side. Moreover, the macroscopic defects already can be found at the unheated side. In detail, voids arise around the unheated side of the sample within the necked region as shown in Fig. 4(b). Such characteristic of the distribution of deformation

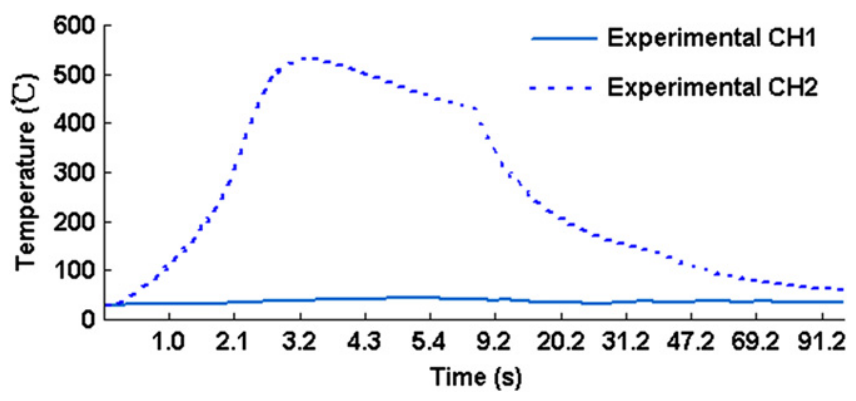

Fig. 2. Temperature histories of the test points $\mathrm{CH} 1$ and $\mathrm{CH} 2$.

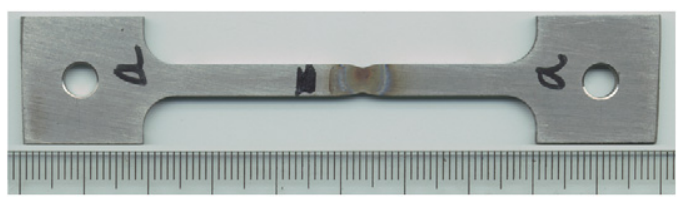

Fig. 3. Global profile of the processed specimens.

Table 1

Temperature dependent mechanical properties.

\begin{tabular}{|c|c|c|c|c|c|c|}
\hline$T\left({ }^{\circ} \mathrm{C}\right)$ & 25 & 93 & 205 & 315 & 427 & 538 \\
\hline$\alpha\left(\times 10^{-6} \mathrm{~K}^{-1}\right)$ & 4.90 & 5.0 & 5.18 & 5.35 & 5.52 & 5.69 \\
\hline$E(\mathrm{GPa})$ & 110 & 105 & 99.1 & 93.3 & 86.9 & 70.6 \\
\hline$\sigma_{s}(\mathrm{MPa})$ & 916 & 827 & 695 & 594 & 480 & 313 \\
\hline$\varepsilon_{s}\left(\times 10^{-3}\right)$ & 8.33 & 7.88 & 7.01 & 6.37 & 5.52 & 4.43 \\
\hline$E_{T}(\mathrm{GPa})$ & 35.5 & 30.2 & 30.2 & 32.3 & 34.5 & 31.2 \\
\hline$v$ & 0.3475 & 0.3515 & 0.3575 & 0.3545 & 0.369 & 0.375 \\
\hline
\end{tabular}



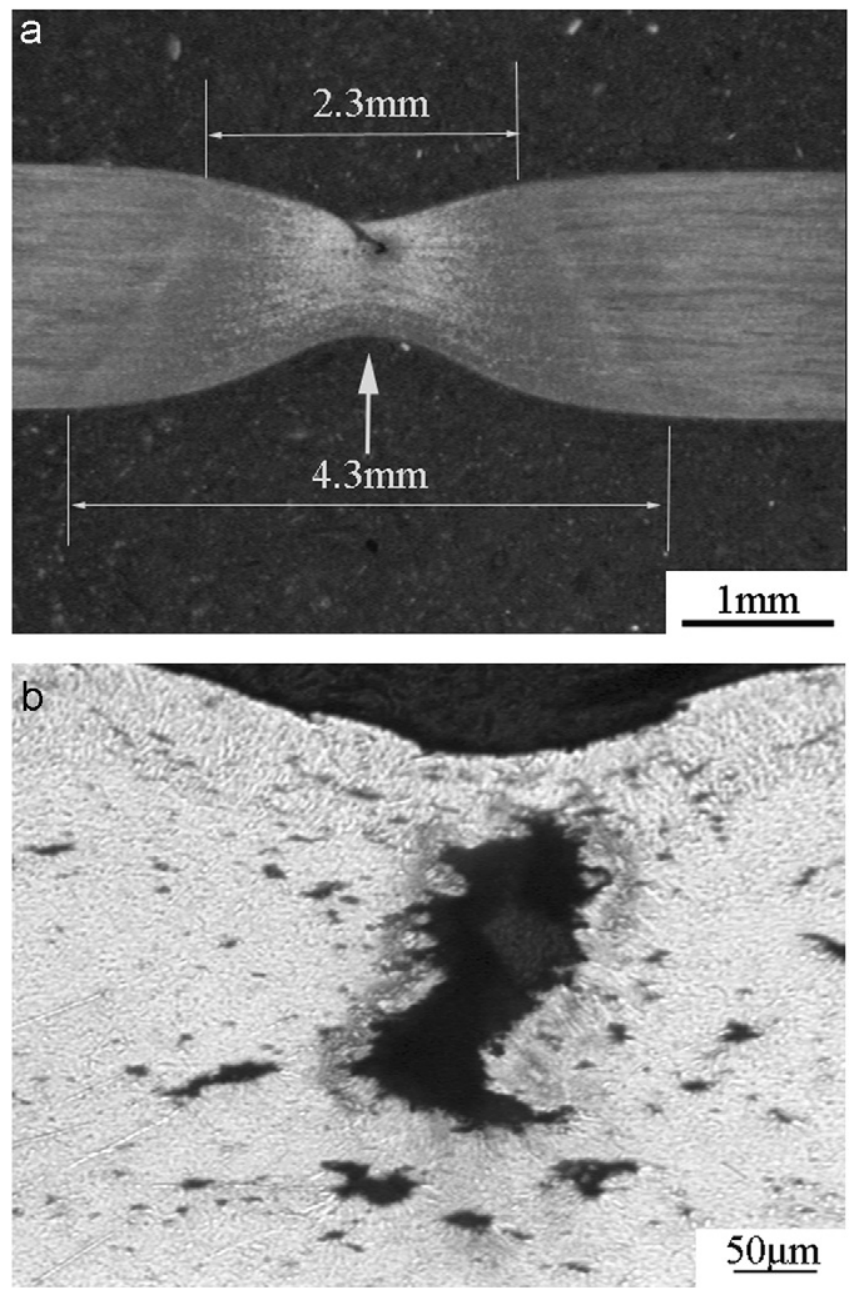

Fig. 4. (a) Necked morphology and (b) defects arising in the necking region.

and defects also show partly that the elevated temperature will enhance the ductility of the material to some extent. Fig. 5(a) provides the micro-structures of the material within the strain localized zone, which indicates some martensites resulted from the phase transformation under the action of stress and heating. In comparison, the initial state of the rolled Ti6Al4V alloy is shown in Fig. 5(b), which is composed of $\alpha$ and $\beta$ phases.

It is noteworthy that Nemat-Nasser et al. [21] studied the dynamic responses of pre-heated Ti6Al4V alloys and found that the flow stress is very sensitive to the temperature of the material, which can partly explain the plastic concentration in the present experiments. That is, the local temperature elevation decreases the yield stress of the material therein and leads to the plastic strain. Moreover, the stiffness will also be reduced with increasing the temperature, which will increase the strain therein through redistributing the stain field within the specimen. In effect, such strain redistribution will further enhance the deformation concentration.

\section{Numerical computation of the temperature}

To describe the temperature pattern of the strip subjected to localized heating, a finite element model as shown in Fig. 6 was set $\mathrm{p}$. Considering the fact that the temperature at the position around the oblique shoulder of the specimen is nearly same as the ambient temperature, only the effective strip is modeled. Furthermore, the quarter model is adopted in the computation because of
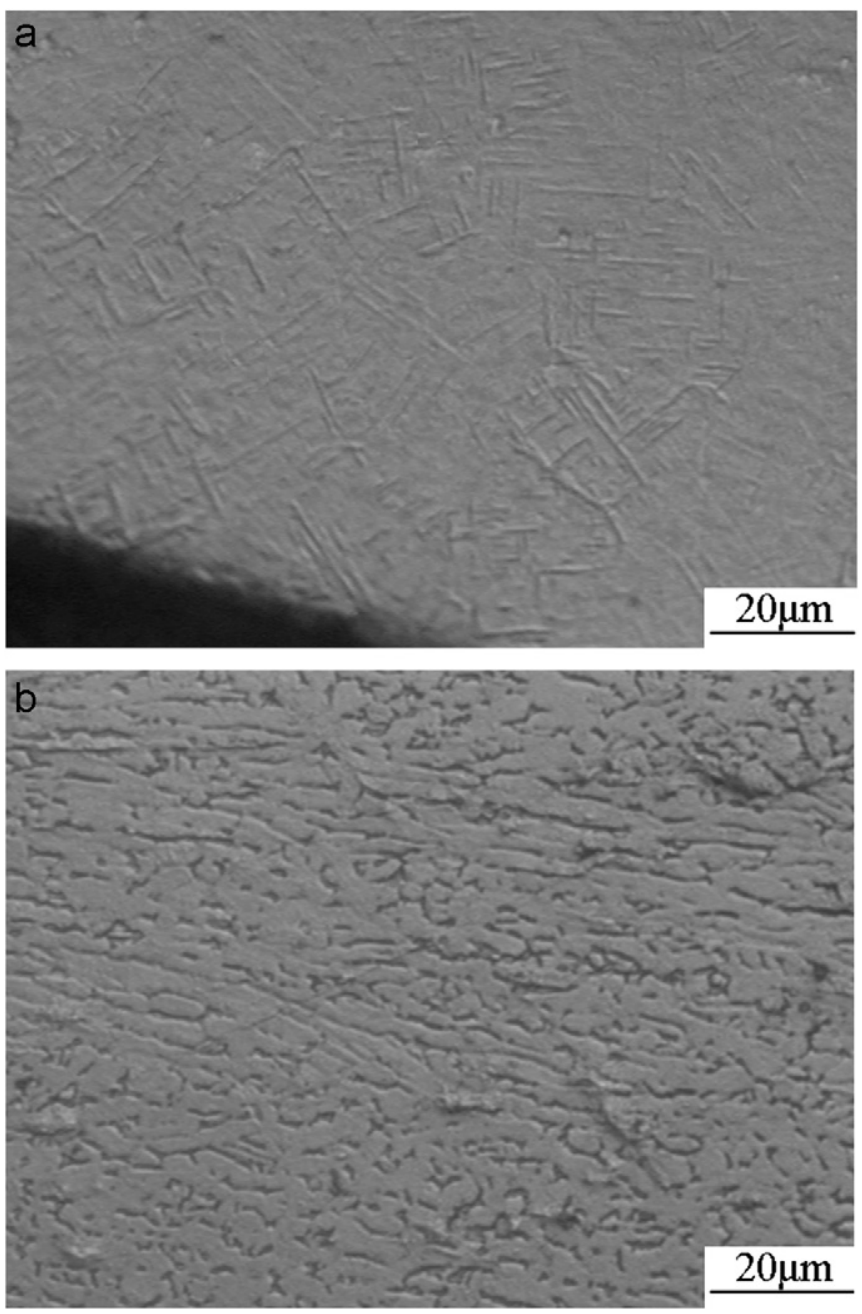

Fig. 5. Micro-structures of (a) tested and (b) origin materials.

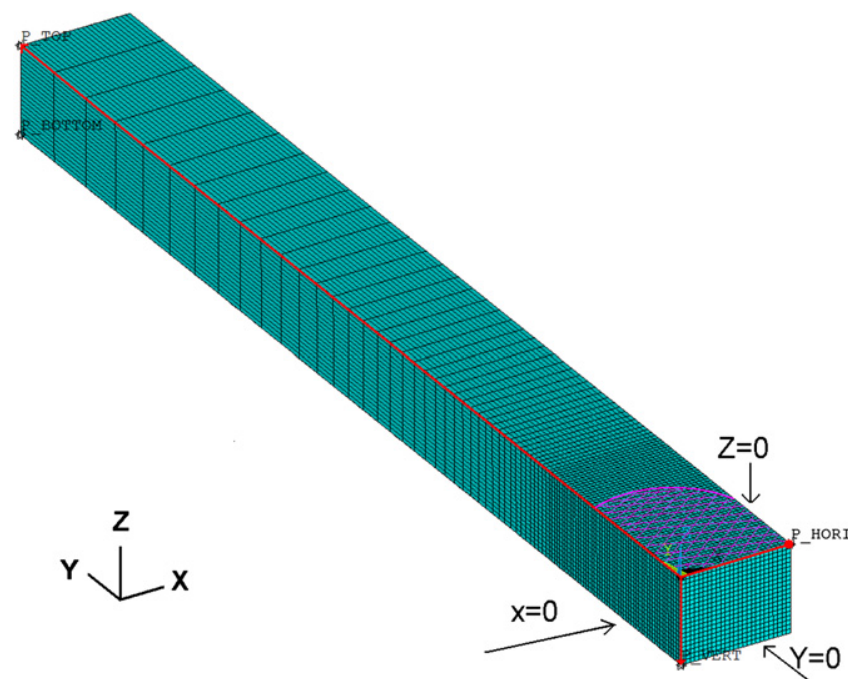

Fig. 6. Finite element model of the quarter geometry.

the symmetries of the sample and the loadings. Four paths for mapping the results are denoted in Fig. 6, where the paths p_top on the top surface and p_bottom on the bottom surface are along the length direction, while the paths p_hori and p_vert are along the width direction and depth direction, respectively. 
The heat conduction equation within the sample is

$\frac{\partial^{2} T}{\partial x^{2}}+\frac{\partial^{2} T}{\partial y^{2}}+\frac{\partial^{2} T}{\partial z^{2}}=\frac{\rho c}{k} \frac{\partial T}{\partial t}$,

with the thermal boundary condition at the region irradiated by Laser beam on surface $z=0$ is

$-k \frac{\partial T}{\partial n}=q$,

the symmetric boundary conditions at the surfaces $y=0$ and $x=0$ are

$k \frac{\partial T}{\partial n}=0$,

and the thermal boundary condition at the other surface regions of the sample is

$k \frac{\partial T}{\partial n}=h\left(T_{e}-T\right)$

where $T$ represents temperature of the materials, $\rho(=4470 \mathrm{~kg} /$ $\left.\mathrm{m}^{3}\right)$ is the density of the material, $q\left(=1.70 \mathrm{MW} / \mathrm{m}^{2}\right)$ the heat flux exerted on the irradiated circular region of radius $3 \mathrm{~mm}, h$ $\left(=25 \mathrm{~W} / \mathrm{m}^{2}{ }^{\circ} \mathrm{C}\right)$ the heat transfer coefficient between the solid surface and the cooling liquid, $T_{e}\left(=25^{\circ} \mathrm{C}\right)$ ambient temperature

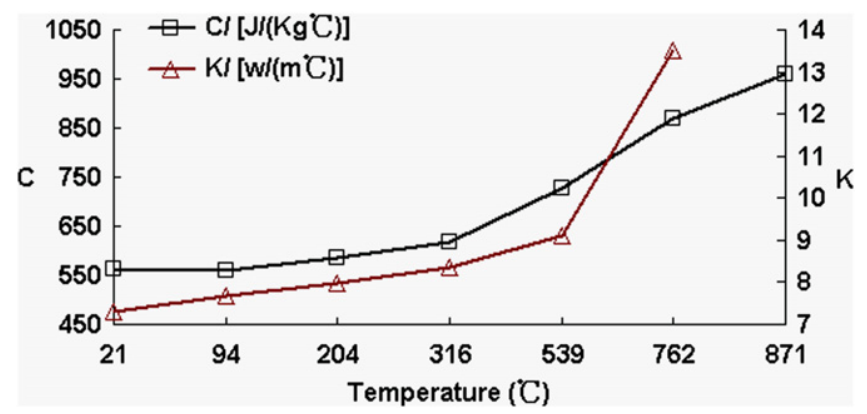

Fig. 7. Temperature dependent physical properties.
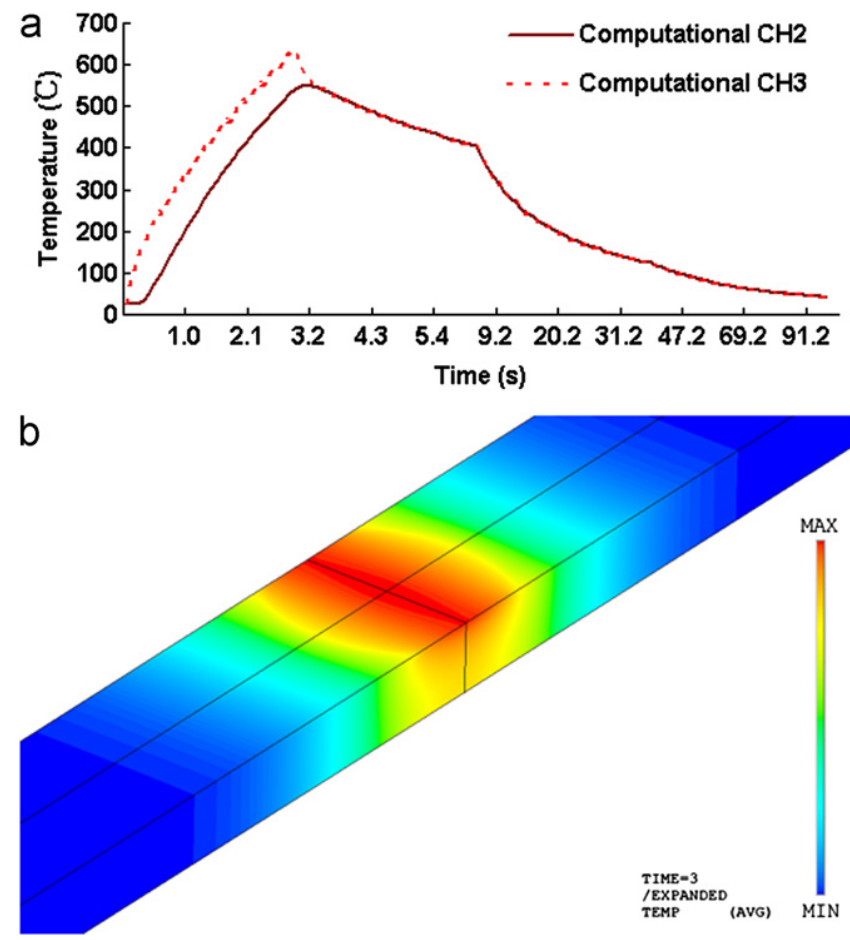

Fig. 8. (a) Temperature histories for points $\mathrm{CH} 2$ and $\mathrm{CH} 3$ and (b) temperature contour at $t=3 \mathrm{~s}$. and $n$ represents the normal to the surface. The heat capacity $c$ and thermal conductivity $k$ of the material are plotted in Fig. 7.

After solving the heat conduction Eq. (1) with boundary conditions (2), (3) and (4), the temperature histories of the two test points $\mathrm{CH} 2$ and $\mathrm{CH} 3$ are graphed in Fig. 8(a), for which the loci are displayed in Fig. 1(b). The temperature contour of the strip at the instant when time $t=3 \mathrm{~s}$ is shown in Fig. 8(b), which shows that the temperature elevation is largely concentrated around the heated region.

For comparison, the computational and experimental temperature histories of the test point $\mathrm{CH} 2$ are plotted in Fig. 9, which shows that the theoretical results are almost in accordance to the experimental results with only a little bit difference in the temperature elevating stage. Such difference may be due to the presumed constant absorption coefficient of the Laser beam radiation used in computation for the sample material, which is actually temperature dependent.

The spatial distributions of the temperature in the strip are shown in Figs. 10, 11 and 12. Fig. 10 shows the length direction

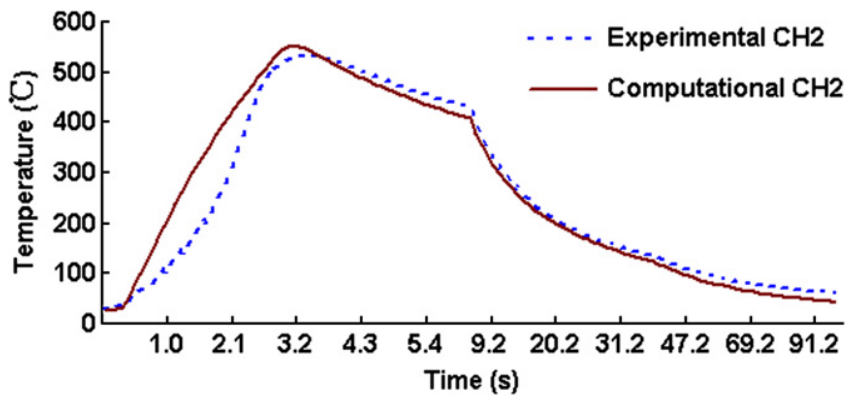

Fig. 9. Computational and experimental results of temperature for point $\mathrm{CH} 2$.

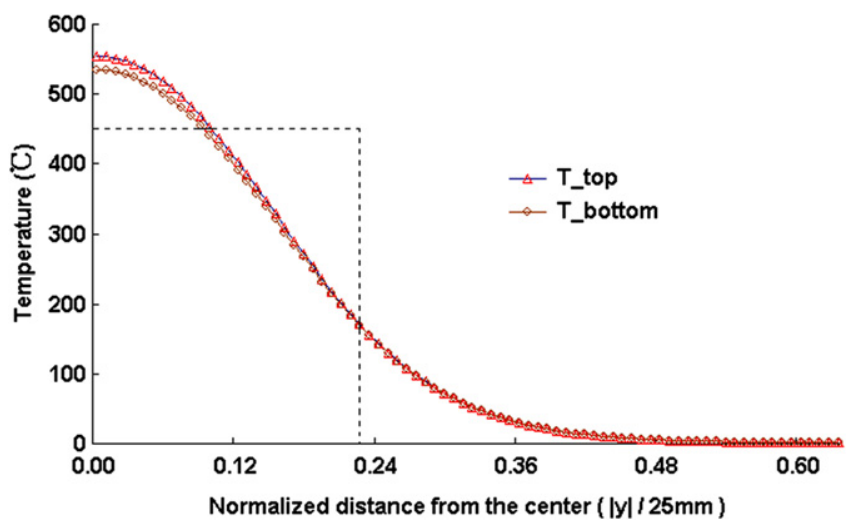

Fig. 10. Temperature profile along the length-oriented path at $t=3 \mathrm{~s}$.

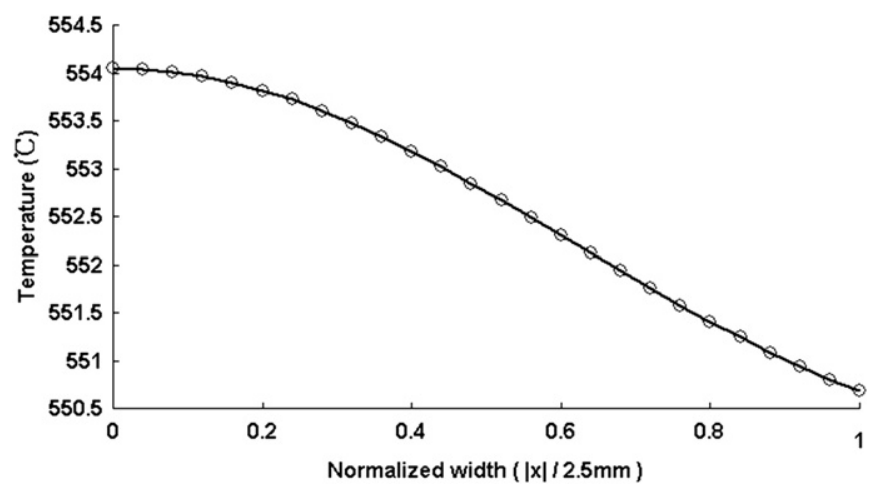

Fig. 11. Temperature profile along the width-oriented path at $t=3 \mathrm{~s}$. 


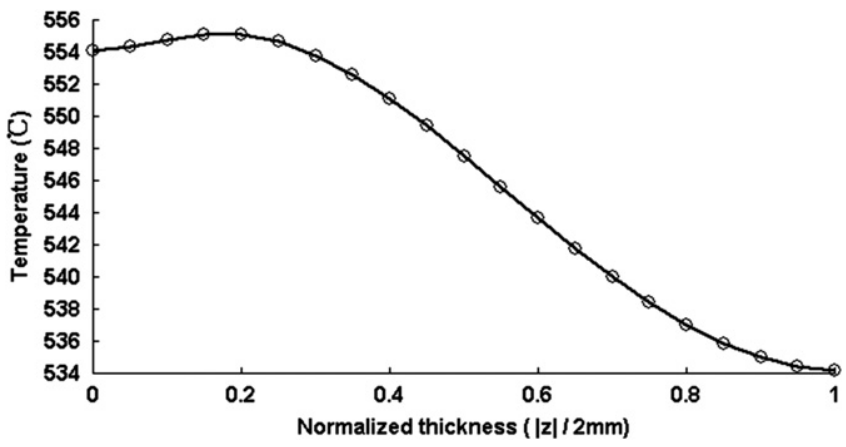

Fig. 12. Temperature profile through the thickness at $t=3 \mathrm{~s}$.

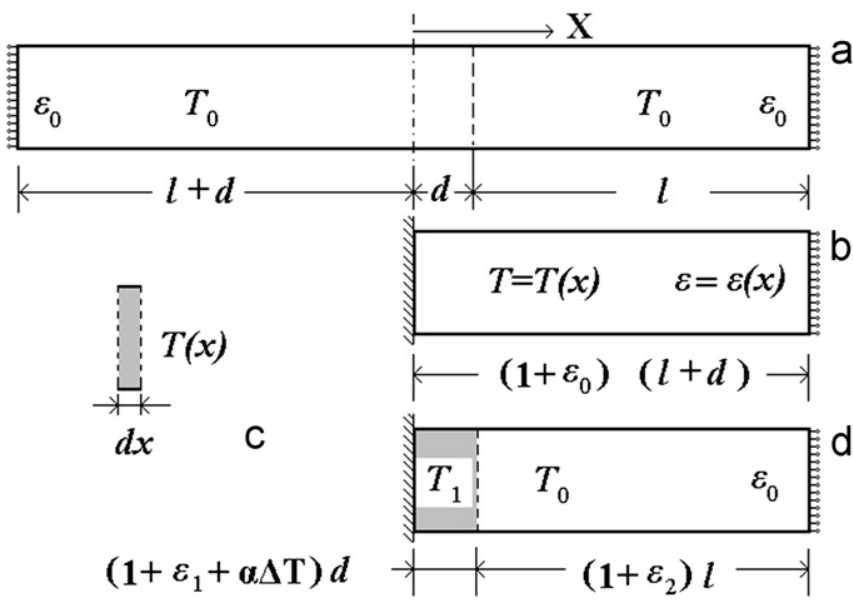

Fig. 13. Sketch of the deformation of the strip.

distribution of the temperature, in which the curves titled by T_top and T_bottom represent the temperature distribution along the paths at the top surface radiated by the Laser beam and the bottom surface, respectively. It is indicated in Fig. 10 that the high temperature elevation is largely concentrated on the heated segment as confined by the dashed lines. Figs. 11 and 12 show the width direction and depth direction distributions of the temperature, respectively. One can see in Figs. 11 and 12 that the temperature is almost uniform along the width or depth direction.

\section{Theoretical formulation of the deformation}

As a preliminary study, the plastic deformation induced by localized temperature elevation in a pre-stressed strip is investigated with the one dimensional model as shown in Fig. 13, in which the total length of the strip under consideration is $(l+d)$. Here, $d$ represents the heated segment length and $l$ the unheated segment length. The elastic modulus and Poisson's ratio of the material at ambient temperature $T_{0}$ are denoted by $E_{0}$ and $v_{0}$, respectively. Initially, the strip is uniformly stretched to the state of axial strain $\varepsilon_{0}$, which is assured to be lower than the yield strain of the material $\varepsilon_{0 s}$ at ambient temperature.

When the elastically stretched strip is heated to a state of nonuniform temperature distribution as shown in Fig. 13(b), the strain shall be redistributed throughout the specimen due to the softening and expanding of the materials in the heated region. The deformation along X-direction of the infinitesimal segment as shown in Fig. 13(c) can be written as

$\left(\varepsilon(x)+\alpha\left(T(x)-T_{0}\right)\right) d x$,
From the condition of constrained total displacement, we have the geometrical equation

$\int_{0}^{l+d}\left(\varepsilon(x)+\alpha\left(T(x)-T_{0}\right)\right) d x=\Delta l$

The total axial deformation of the strip is

$\Delta l=\varepsilon_{0}(l+d)=\varepsilon_{0} l\left(1+r_{d l}\right)$,

with

$r_{d l}=d / l$

It is assumed that the segment of length $2 d$ is uniformly heated up to $T_{1}$.

Denote Young's modulus and Poisson's ratio of the material for temperature $T_{1}$ as $E_{1}$ and $v_{1}$, respectively. Besides, the relationship between the yield stress $\sigma_{s 1}$ and yield strain $\varepsilon_{s 1}$ can be written approximately as

$\sigma_{s 1}=E_{1} \varepsilon_{s 1}$

at certain elevated temperature condition if the effect of the triaxial stresses is ignored for simplicity. The bi-linear isotropic hardening modeling is assumed for the material the tangent modulus of the material is denoted as $E_{T 1}$.

Let the average strain of the heated segment be $\varepsilon_{1}$ and that of the unchanged part be $\varepsilon_{2}$, by considering the fact that both sides of the strip are fixed after being stretched, one can get quickly the following geometry equation

$\Delta l=\left(r_{d l} \varepsilon_{1}+r_{d l} \alpha \Delta T+\varepsilon_{2}\right) l$,

where $\alpha$ is the thermal expansion coefficient and

$\Delta T=T_{1}-T_{0}$

At the same time, the tension equilibrium across the interface between the heated segment and the unchanged segment requires that

$\sigma_{1}\left(1-\varepsilon_{1} v_{1}\right)^{2}(1+\alpha \Delta T)^{2}=\sigma_{2}\left(1-\varepsilon_{2} v_{0}\right)^{2}$

If the items of higher orders are ignored, the Eq. (12) will reduce to

$\sigma_{1} \approx \sigma_{2}$

For a while, one can assume ideal elastic behavior for both the heated region and unheated region, then (13) can be expressed as $E_{1} \varepsilon_{1} \approx E_{0} \varepsilon_{2}$

Combining the Eqs. (7), (10) and (14), one can get

$\varepsilon_{1} \approx\left(\varepsilon_{0}\left(1+r_{d l}\right)-r_{d l} \alpha \Delta T\right) /\left(r_{d l}+\left(E_{1} / E_{0}\right)\right)$

Now, we can justify whether the assumption of ideal elastic behavior is matched by comparing the magnitude of $\varepsilon_{1}$ to the yield strain of the materials at that temperature, i.e. $\varepsilon_{s 1}$. That is, if $\varepsilon_{1} \leq \varepsilon_{s 1}$,

then the full strain of the heated segment is already obtained as (15), which means no plastic strain arises. Or else, if

$\varepsilon_{1}>\varepsilon_{s 1}$,

Then the equilibrium Eq. (14) should be extended as

$\varepsilon_{s 1} E_{1}+\left(\varepsilon_{1}-\varepsilon_{s 1}\right) E_{T 1} \approx \varepsilon_{2} E_{0}$

Thus, combining the Eqs. (7), (10) and (18), one can get

$\varepsilon_{1} \approx\left(\varepsilon_{0}\left(1+r_{d l}\right)-r_{d l} \alpha \Delta T-\varepsilon_{s 1}\left(E_{1} / E_{0}\right)\left(1-E_{T 1} / E_{1}\right)\right) /\left(r_{d l}+\left(E_{T 1} / E_{0}\right)\right)$

And the plastic strain, $\varepsilon_{1 p}$ of the heated segment can be obtained quickly as

$\varepsilon_{1 p}=\left(\varepsilon_{1}-\varepsilon_{s 1}\right)\left(1-E_{T 1} / E_{1}\right)$, 
which can be written in detail as

$\varepsilon_{1 p} \approx\left(\varepsilon_{0}\left(1+r_{d l}\right)-r_{d l} \alpha \Delta T-\varepsilon_{s 1}\left(E_{1} / E_{0}+r_{d l}\right)\right)\left(1-E_{T 1} / E_{1}\right) /\left(r_{d l}+E_{T 1} / E_{0}\right)$

Again, from the inequality (16), we can get the condition under that no plastic deformation will arise in the strip, that is

$\varepsilon_{0}\left(1+r_{d l}\right) \leq r_{d l} \alpha \Delta T+\varepsilon_{s 1}\left(r_{d l}+\left(E_{1} / E_{0}\right)\right)$

Of course, the condition inequality (22) can also be derived from (21) by letting the item in the first bracket not greater than zero.

Further, the condition inequality (22) can be written as

$r_{d l}\left(\varepsilon_{0}-\alpha \Delta T-\varepsilon_{s 1}\right) \leq\left(\varepsilon_{s 1}\left(E_{1} / E_{0}\right)-\varepsilon_{0}\right)$

Which means there should be several cases to be categorized as follows.

$\varepsilon_{0} \leq \varepsilon_{s 1}\left(E_{1} / E_{0}\right)$

which means that the (23) is definitely satisfied as considering the fact that $E_{1} \leq E_{0}$ due to thermal softening of the common material. Thus, there exists a maximum for the initial elastic

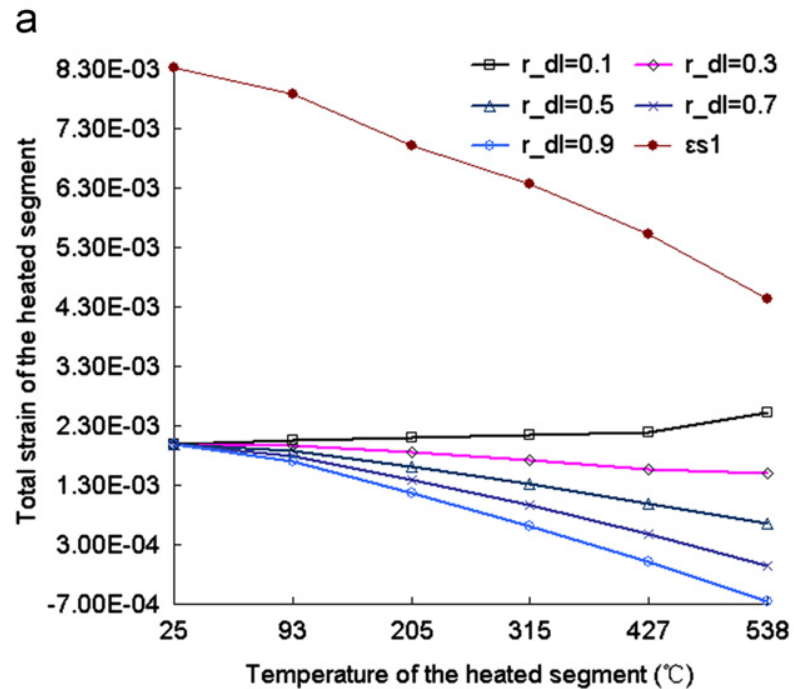

C

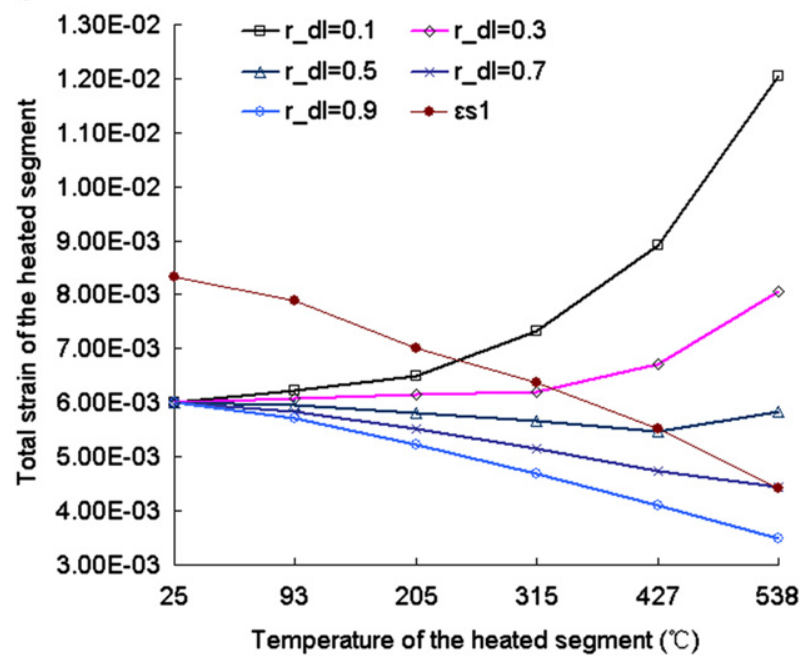

strain to ensure no plastic deformation will be developed by the subsequent heating.

$\varepsilon_{s 1}\left(E_{1} / E_{0}\right) \leq \varepsilon_{0} \leq\left(\alpha \Delta T+\varepsilon_{s 1}\right)$,

under which plastic deformation will arise after being locally heated if

$r_{d l}<\left(\varepsilon_{s 1}\left(E_{1} / E_{0}\right)-\varepsilon_{0}\right) /\left(\varepsilon_{0}-\alpha \Delta T-\varepsilon_{s 1}\right)$

$\left.\alpha \Delta T+\varepsilon_{s 1}\right) \leq \varepsilon_{0}$

which means that the (23) is definitely not satisfied. Therefore, plastic deformation will arise no matter how large is the magnitude of $r_{d l}$.

To take an example, the strain of the titanium alloy strip of the thermo-physical parameters as listed in Table 1 can be computed by (15), (19) and (21). The total effective length of the studied sample is $25 \mathrm{~mm}$, for which the results for different heat segment lengths, different initial strain levels and different elevated temperatures are discussed as shown in Fig. 14(a)-(d) and Fig. 15. In these figures, the symbol $r_{d l}$ represents the parameter

b

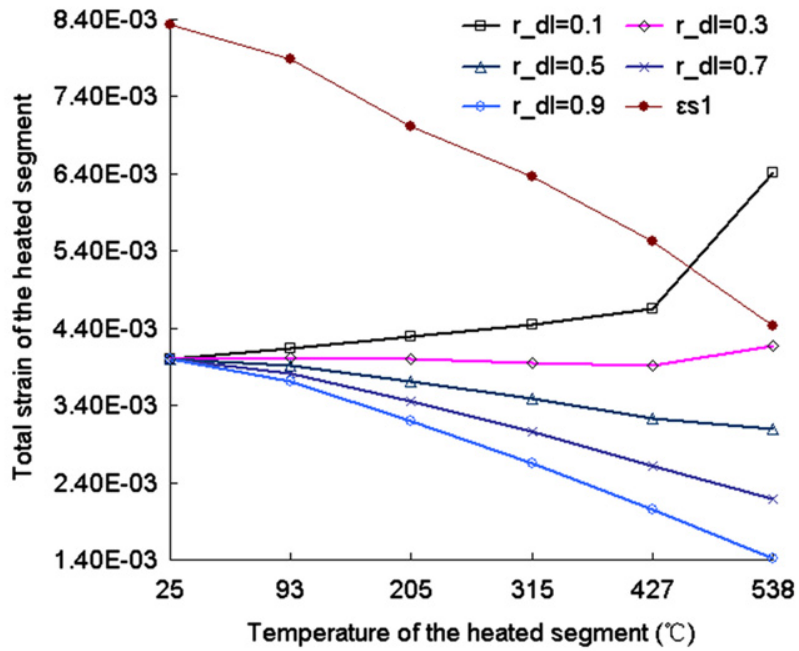

d

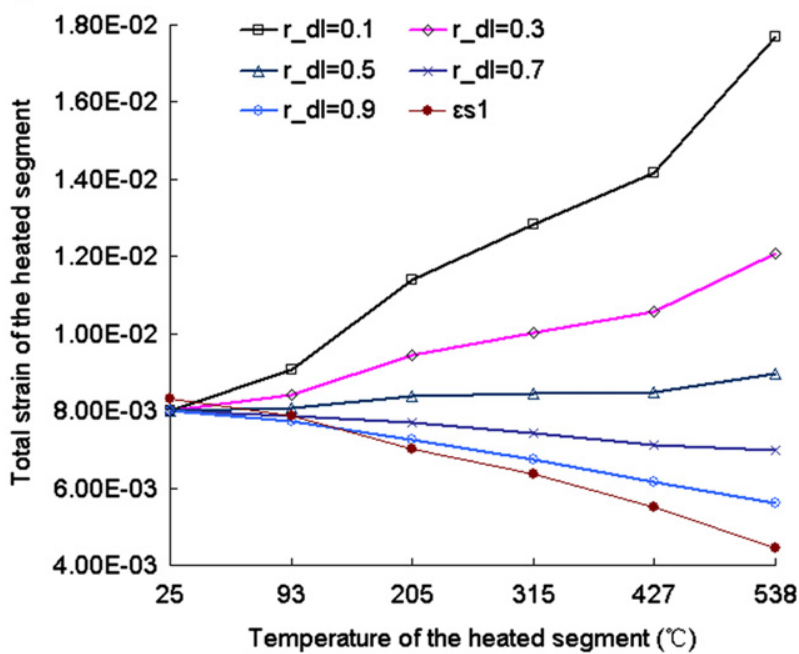

Fig. 14. Total strain of the heated segment versus different elevated temperature for the cases of pre-strain (a) $2 e-3$, (b) $4 e-3$, (c) $6 e-3$ and (d) $8 e-3$. 


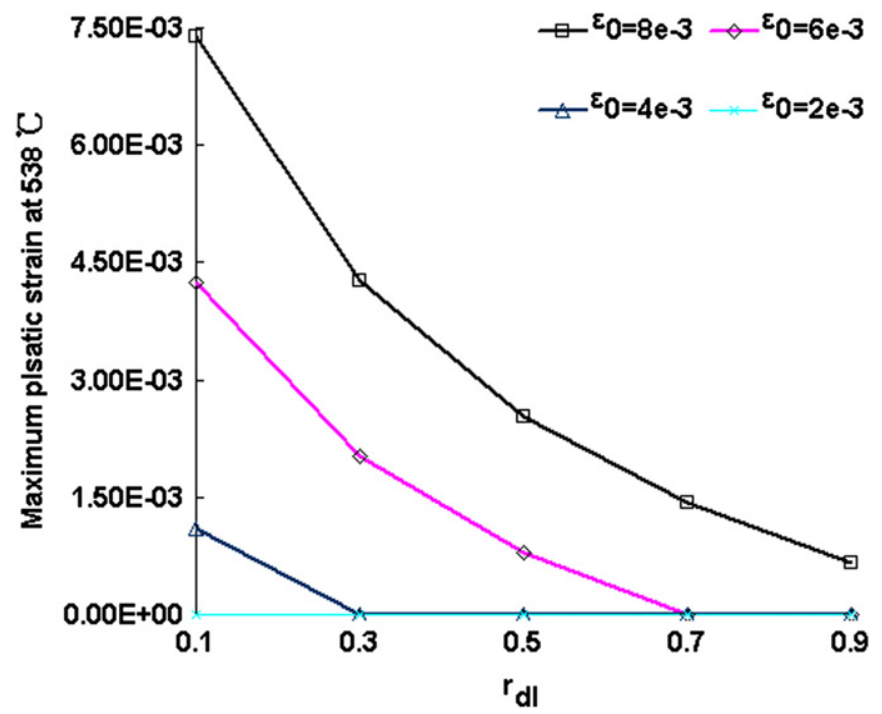

Fig. 15. Maximum plastic strain of the heated segment versus different elevated temperature.

$r_{d l}=d / l, \varepsilon_{s 1}$ represents the temperature dependent yield strain of the materials and $\varepsilon_{0}$ is the initial elastic strain of the strip.

By comparing Fig. 14(a)-(d), one can find that the total strain of the heated segment increases with increasing the initial elastic strain of the sample. In particular, the total strain of the heated segment will never exceed the yield strain even the elevated temperature is $538{ }^{\circ} \mathrm{C}$ if the initial strain is $2 \mathrm{e}-3$ as shown in Fig. 14(a), which means no plastic strain will be developed. Furthermore, it is indicated that the total strain of the heated segment increases quickly with decreasing the ratio of the heated segment length to that of the whole effective length of the strip. It seems like that the total strain of the heated segment will be always greater than the initial strain if the heated segment is short enough.

The maximum plastic strain magnitudes of the strip heated up to about $538{ }^{\circ} \mathrm{C}$ for the cases of different combinations of initial strain and heated length are shown in Fig. 15. It is indicated again that decreasing the heated length or increasing the initial strain will increase the maximum plastic strain. Moreover, no plastic strain appears if the initial strain is $2 \mathrm{e}-3$ or smaller.

It can be understood easily that once the plastic strain approaches to some critical value, damage will be developed extensively and the deformation stability will be disturbed. The instability of the plastic deformation will lead to the necking of the strip ultimately, which was validated by the experimental results aforementioned.

\section{Conclusions}

Experimental results reveal that the non-uniform temperature elevation will re-distribute the strain in the as-stretched strip and strain will largely concentrate on the heated region due to the thermal softening of the material therein.

Theoretical results further reveal that the magnitude of the final plastic strain depends largely on the initial elastic strain. In detail, if an initially elastically stretched strip is partially heated up to elevated temperature $T_{1}$, under which the elastic modulus and yield strain are $E_{1}$ and $\varepsilon_{s 1}$, respectively, then there exist three typical cases as follows.

(1) No plastic strain will arise in the pre-elastic-stretched strip if the initial elastic strain is less than the lower limit, i.e., $\varepsilon_{0} \leq \varepsilon_{s 1}\left(E_{1} / E_{0}\right)$
(2) Plastic strain will definitely arise if the initial elastic strain is greater than the upper limit, i.e., $\left(\alpha \Delta T+\varepsilon_{s 1}\right) \leq \varepsilon_{0}$;

(3) Plastic strain will arise when the length of the heated segment is small enough, i.e., $r_{d l}<\left(\varepsilon_{s 1}\left(E_{1} / E_{0}\right)-\varepsilon_{0}\right) /\left(\varepsilon_{0}-\alpha \Delta T-\varepsilon_{s 1}\right)$, if the initial elastic strain falls into the range between the lower and upper limits, i.e., $\varepsilon_{s 1}\left(E_{1} / E_{0}\right) \leq \varepsilon_{0} \leq\left(\alpha \Delta T+\varepsilon_{s 1}\right)$. Moreover, the plastic strain magnitude of the heated segment will increase with increasing the initial elastic strain or decreasing the length of the heated segment.

\section{Acknowledgments}

This work was supported by the National Natural Science Foundation of China (Grant nos. 10872199, 11002145 and 50901084). The authors also thank Mrs. Guo X.L. and Mr. Xiao J.H. for their assistances in conducting the experiments.

\section{References}

[1] Lee K-C, Lin J. Transient deformation of thin metal sheets during pulsed laser forming. Optics and Laser Technology 2002;34:639-48.

[2] Shi Y, Liu Y, Yi P, Hu J. Effect of different heating methods on deformation of metal plate under upsetting mechanism in laser forming. Optics and Laser Technology 2012;44:486-91.

[3] Shinho K, Tokuo A. Bending of pipe having nonuniform distribution of temperature by high-frequency induction heating. Journal of the Japan Society for Technology of Plasticity 2001;42:139-44.

[4] Shen ZH, Zhang SY, Lu J, Ni XW. Mathematical modeling of laser induced heating and melting in solids. Optics and Laser Technology 2001;33:533-7.

[5] Yilbas BS, Al-Aqeeli N. Analytical investigation into laser pulse heating and thermal stresses. Optics and Laser Technology 2009;41:132-9.

[6] Peng Q, Chen G-N, Wu C-W, Luo G-X, Wang X-F. Laser-assisted pre-stress forming for integral panels. Acta Aeronautica et Astronautica Sinica 2009;30: 1544-8.

[7] Peng Q, Chen G-N, Wang M-X, Wu C-W. Effects of process parameters on efficiency of Laser-assisted pre-stress forming. Chinese Journal of Lasers 2011;38:1003006.

[8] Tana KH, Yuan WF. Buckling of elastically restrained steel columns under longitudinal non-uniform temperature distribution. Journal of Constructional Steel Research 2008;64:51-61.

[9] Xia Y, Xu Y-L, Wei Z-L, Zhu H-P, Zhou X-Q. Variation of structural vibration characteristics versus non-uniform temperature distribution. Engineering Structures 2011;2011(33):146-53.

[10] Doghri I, Billardon R. Investigation of localization due to damage in elastoplastic materials. Mechanics of Materials 1995;19:129-49.

[11] Haddag B, Abed-Meraim F, Balan T. Strain localization analysis using a large deformation anisotropic elastic-plastic model coupled with damage. International Journal of Plasticity 2009;25:1970-96.

[12] Kobayashi M. Analysis of deformation localization based on proposed theory of ultrasonic wave velocity propagating in plastically deformed solids. International Journal of Plasticity 2010;26:107-25.

[13] Bychkov AA, Karpinskii DN. Numerical analysis of necking conditions in a thermoviscoplastic rod in tension. Journal of Applied Mechanics and Technical Physics 1998;39:634-8.

[14] Elfmark J. Development of deformation instability in hot tensile test specimens. Metallurgical Transactions A 1991;22:2297-302.

[15] Bychkov AA, Karpinskii DN. Analysis of the conditions of necking in a porous rod subjected to tension. Strength of Materials 1999;30:274-81.

[16] Bychkov AA, Karpinskii DN. Effect of an electric current on necking in a tensile rod. Journal of Applied Mechanics and Technical Physics 1999;40:930-5.

[17] Fressengeas C, Molinari A. Inertia and thermal effects on the localization of plastic flow. Acta Metallurgica 1985;33:387-96.

[18] Benallal A, Bigoni D. Effects of temperature and thermo-mechanical couplings on material instabilities and strain localization of inelastic materials. Journal of the Mechanics and Physics of Solids 2004;52:725-53.

[19] Fukuhara M, Sanpei A. Elastic moduli and internal frictions of Inconel 718 and Ti-6Al-4V as a function of temperature. Journal of Materials Science Letters 1993;12:1122-4.

[20] Knovel Interactive Edition, Military Handbook-MIL-HDBK-5H: Metallic materials and elements for aerospace vehicle structures; Interactive Graphs for 5.4.1 Ti-6Al-4V; 2001.

[21] Nemat-Nasser S, Guo W-G, Nesterenko VF, Indrakanti SS, Gu Y-B. Dynamic response of conventional and hot isostatically pressed $\mathrm{Ti}-6 \mathrm{Al}-4 \mathrm{~V}$ alloys: experiments and modeling. Mechanics of Materials 2001;33:425-39. 